\title{
Mejores Prácticas de Desarrollo Profesional Docente en Estados Unidos
}

\author{
Laura M. DESIMONE* \& Michael S. GARET**
}

(*) University of Pennsylvania, (**) American Institutes for Research (Estados Unidos de América)

(Recibido el 6 de febrero de 2015; Aceptado el 6 de septiembre de 2015)

RESUMEN: Este artículo analiza las mejores prácticas de desarrollo profesional docente (DPD) en los Estados Unidos de América (USA). Comenzamos presentando un marco conceptual sobre DPD, que sugiere la existencia de cinco características clave que favorecen un DPD efectivo: foco en el contenido, aprendizaje activo, coherencia, duración sostenida, y participación colectiva. Continuamos describiendo los resultados de recientes investigaciones que han puesto a prueba dichas cinco características en USA, enfatizando los resultados de rigurosos estudios experimentales aleatorizados. Discutimos las lecciones aprendidas a partir de dichos estudios, que nos han permitido refinar nuestro marco teórico sobre el DPD efectivo. Hemos aprendido que (a) cambiar el comportamiento de los profesores en clase es más fácil que mejorar sus conocimientos disciplinares o las estrategias instruccionales de alto nivel; (b) los profesores varían en respuesta al mismo DPD; (c) DPD es más exitoso cuando está explícitamente conectado con la práctica docente; (d) la investigación y la implementación del DPD debe tener en cuenta las condiciones de las áreas urbanas (e.g., la movilidad de estudiantes y profesores); y (e) los líderes juegan un rol fundamental apoyando y animando a los profesores para que apliquen en sus clases las ideas y estrategias aprendidas en DPD. Después examinamos tres tendencias generales en la evaluación reciente del DPD en USA - un descenso en el uso de cursos y talleres de corta duración, la conexión del DPD con las evaluaciones del profesorado, y el uso de video-tecnología para mejorar y supervisar los efectos del DPD. Finalmente, discutimos las dificultades que los distritos y las escuelas encuentran para implementar DPD efectivo.

Palabras clave: desarrollo profesional del profesorado, DPD de alta calidad, estudios causales de DPD, características del DPD efectivo

\section{Best Practices in Teachers' Professional Development in the United States}

ABSTRACT: This paper discusses best practices in teachers' professional development (PD) in the United States (U.S.). We begin by presenting a conceptual framework for effective 
professional development, which suggests five key features that make professional development effective-content focus, active learning, coherence, sustained duration, and collective participation. We then describe the findings from recent U.S. research that has tested the five features, with an emphasis on the results of rigorous randomized control trials. We discuss several insights gained from this work and that have helped refine the framework. They are that (a) changing procedural classroom behavior is easier than improving content knowledge or inquiry-oriented instruction techniques; (b) teachers vary in response to the same PD; (c) PD is more successful when it is explicitly linked to classroom lessons; (d) PD research and implementation must allow for urban contexts (e.g., student and teacher mobility); and (e) leadership plays a key role in supporting and encouraging teachers to implement in the classroom the ideas and strategies they learned in the PD. We then examine three major trends in how professional development for teachers is evolving in the U.S. - a move away from short workshops, linking teacher PD to evaluations, and the use of video technology to improve and monitor the effects of PD. Finally, we discuss the challenges faced by districts and schools in implementing effective professional development.

Key words: teachers' professional development; high-quality professional development; causal studies of professional development; features of effective professional development

Correspondencia: Laura M. Desimone, Graduate School of Education/ University of Pennsylvania. Phone: 215-746-5699. Email: lauramd@gse.upenn.edu

\section{Cómo citar este artículo}

Desimone, L. M., \& Garet, M. S. (2015). Mejores prácticas de desarrollo profesional docente en Estados Unidos (trad. al castellano de A. Bautista). Psychology, Society and Education, 7(3), 356-369. [V.O.: Best practices in teachers' professional development in the United States. Psychology, Society and Education, 7(3), 252-263].

\section{Introducción}

Este artículo analiza las mejores prácticas de desarrollo profesional docente (DPD) en los Estados Unidos de América (USA). Comenzamos presentando un marco conceptual sobre DPD, que sugiere la existencia de cinco características clave que hacen el DPD efectivo. Continuamos describiendo los resultados de recientes investigaciones que han puesto a prueba dichas cinco características en USA. Discutimos las lecciones aprendidas a partir de dichos estudios, que nos han permitido refinar distintos aspectos de nuestro marco teórico. Después examinamos tres tendencias generales en la evolución reciente del DPD en USA, y discutimos las dificultades que los distritos y las escuelas encuentran para implementar DPD efectivo.

\section{Un Marco Conceptual sobre el Desarrollo Profesional Docente Efectivo}

Existe considerable evidencia en estudios realizados en USA de que para que el DPD sea eficaz en términos de mejorar las prácticas docentes y el aprendizaje de los alumnos, al menos cinco características necesitan estar presentes: (a) foco en el contenido: las 
actividades del DPD necesitan estar centradas en el contenido que se enseña y en cómo los estudiantes aprenden dicho contenido; (b) aprendizaje activo: los profesores necesitan tener oportunidades para observar, recibir feedback, analizar el trabajo de los estudiantes, o presentar su propio trabajo, en vez de escuchar clases o conferencias de forma pasiva; (c) coherencia: el contenido, las metas, y las actividades del DPD necesitan ser consistentes con el curriculum y la dirección de las escuelas, con el conocimiento y las creencias de los profesores, con las necesidades de los estudiantes, y con las reformas y políticas a nivel escolar, de distritos y del estado; (d) duración sostenida: para ser efectivo, el DPD necesita desarrollarse durante el curso escolar completo e incluir 20 horas o más de tiempo de contacto con los facilitadores; y (e) participación colectiva: los grupos de profesores que participan en DPD efectivo enseñan la misma asignatura, en el mismo curso y en la misma escuela, lo cual les permite crear comunidades interactivas de aprendizaje (ver Desimone, 2009).

La evidencia que apoya estas cinco características procede de estudios transversales (Garet, Porter, Desimone, Birman, \& Yoon, 2001), longitudinales (e.g., Desimone, Porter, Garet, Yoon, \& Birman, 2002; Desimone, Smith, \& Phillips, 2013), y de revisiones de la literatura de estudios cualitativos y cuasi-experimentales (Desimone, 2009). Asimismo, recientes estudios experimentales aleatorizados han documentado el éxito del DPD que presenta dichas características en diferentes niveles educativos y materias (e.g., Gersten, Dimino, Jayanthi, Kim, \& Santoro, 2010; Penuel, Gallagher, \& Moorthy, 2011).

También son notables, sin embargo, los decepcionantes resultados obtenidos en varios macro-estudios experimentales aleatorizados que han sido realizados para poner a prueba las cinco características (e.g., Garet et al., 2008; Garet et al., 2011). Estos estudios han mostrado que todavía no está claro cómo traducir las cinco características en una práctica efectiva. Es necesario realizar más investigación al respecto. Sin embargo, un análisis cuidadoso de estos estudios experimentales, tanto de los exitosos como de los no exitosos, ofrece evidencias que nos permiten comprender mejor cómo estos principios generales pueden ser transformados en DPD efectivo (ver también Desimone \& Stuckey, 2014).

\section{Utilizando la Evidencia Reciente para Refinar el Marco Conceptual de las Cinco- Características}

A través de un riguroso examen de las investigaciones sobre DPD realizadas en USA, los investigadores hemos aprendido varias lecciones que están siendo utilizadas actualmente para refinar el marco conceptual de las cinco características, de manera que los distritos y a las escuelas puedan poner en práctica las nuevas ideas encontradas. Estas lecciones, que discutimos a continuación, se centran en cinco: (a) cambiar el comportamiento de los profesores en clase es más fácil que mejorar sus conocimientos disciplinares o las estrategias instruccionales orientadas de alto nivel; (b) los profesores varían en respuesta al mismo DPD; (c) DPD es más exitoso cuando está explícitamente 
conectado con la práctica docente; (d) la investigación y la implementación del DPD debe tener en cuenta los contextos urbanos (e.g., la movilidad de estudiantes y profesores); y (e) los líderes juegan un rol fundamental apoyando y animando a los profesores para que apliquen en sus clases las ideas y estrategias aprendidas en DPD. Pese a que la mayoría de la investigación discutida ha sido realizada en USA, los principios generales que presentamos a continuación podrían ser igualmente aplicables en otros países; no existe evidencia que sugiera lo contrario.

\section{Diferencias en el objetivo del DPD}

Cambiar el comportamiento de los profesores en clase es más fácil que mejorar sus conocimientos disciplinares o las estrategias instruccionales de alto nivel. Uno de los resultados es que las conductas discretas de los profesores son más fáciles de cambiar que su conocimiento de contenidos académicos o que enfoques complejos de enseñanza. DPD diseñado para fomentar el uso de tareas claras, simples y directas, como incrementar las referencias a la escritura durante la lectura en voz alta, o presentar actividades diarias de calentamiento, suele ser exitoso y requiere un modesto número de horas (e.g., Piasta et al., 2010; Sailors \& Price, 2010). En contraste, es difícil cambiar el conocimiento disciplinar de los profesores de forma significativa, al igual que promover cambios en conductas sofisticadas como enseñar matemáticas a nivel conceptual. Cambios de esta naturaleza son menos probables, incluso con gran cantidad de DPD que presenta las cinco características arriba descritas (Garet et al., 2010).

Este resultado se relaciona directamente con la idea de que la efectividad del DPD se fundamenta en dos tipos de teorías sobre la enseñanza y el aprendizaje. La primera es una teoría del cambio, o de hasta qué punto el DPD promueve los cambios deseados en el conocimiento y en las prácticas de los profesores. La segunda es una teoría de la enseñanza, o de hasta qué punto los cambios en las prácticas docentes mejoran el aprendizaje de los alumnos. El DPD puede fracasar si cualquiera de estas teorías es errónea - o bien el DPD fracasa en cambiar los métodos de enseñanza existentes, o los nuevos métodos de enseñanza fracasan en mejorar el aprendizaje de los alumnos (Wayne, Yoon, Zhu, Cronen, \& Garet, 2008).

Centrándonos en la teoría del cambio (del profesor), pareciera que ciertos tipos de cambio son más fácil de producir que otros. Específicamente, es más probable que el DPD pueda cambiar ciertos procedimientos usados por los profesores, mientras que es menos probable cambiar su comprensión de contenidos disciplinares específicos o sus habilidades para la práctica reflexiva. Por ejemplo, a los profesores les resulta más fácil modificar secuencias rutinarias particulares (e.g., señalar las imágenes citadas en un texto, o practicar ejercicios de compresión de palabras durante cierto tiempo cada día) (véase Piasta et al., 2010) que fomentar discusiones de alto nivel entre los estudiantes. Este tipo de discusiones requieren un profundo conocimiento del contenido disciplinar y un dominio de estrategias para promover discusiones y conversaciones. Este tipo de 
cambios, por tanto, son más profundos y exigentes y a la vez menos probables (Cohen \& Ball, 1990).

Los profesores encuentran más fácil modificar conductas o rutinas específicas que no requieren nuevos conocimientos. En parte, esto puede ser debido a que las conductas simples no son tan dependientes de la calidad de su implementación. Por ejemplo, si ofrecer a los estudiantes más oportunidades para practicar produce una mejora en el aprendizaje, la naturaleza y calidad de dichas oportunidades podría no importar, sino ser más bien la cantidad de tiempo que los estudiantes practican lo que produzca la mejora. En contraste, la instrucción basada en el cuestionamiento está estrechamente conectada al aprendizaje de los estudiantes, pero lo que produce la mejora es la naturaleza y calidad del cuestionamiento y las discusiones que ocurren en clase, en vez de la cantidad de tiempo utilizado en dicho tipo de instrucción (Smith, Desimone, \& Ueno, 2005). Esto implica que mejorar la calidad de lo que los profesores hacen es más difícil que simplemente incrementar la cantidad de tiempo que emplean en conductas específicas (e.g., practicar conjuntos de problemas).

Alternativamente, centrándonos en la teoría del aprendizaje, podría ser que los comportamientos específicos abordados en el DPD sean palancas más potentes para la mejorar el aprendizaje de los estudiantes, en comparación con incrementar el conocimiento disciplinar de los profesores o las estrategias de enseñanza basadas en preguntas. Pero existe poca evidencia que sugiera que este sea el caso. En contraste, la evidencia sugiere que cuando la implementación es de calidad, las estrategias de enseñanza basadas en preguntas tiene un efecto muy significativo en el aprendizaje de los alumnos. El desafío radica en diseñar DPD sostenible que fomente este tipo de instrucción de forma efectiva.

\section{Los profesores responden de forma diferente al mismo DPD}

Un segundo resultado de la investigación reciente en USA es que los profesores varían considerablemente en sus respuestas al mismo DPD. Por su parte, esto crea variaciones en los resultados de los alumnos. Los profesores vienen a DPD con diferentes niveles de experiencia y conocimiento del contenido, procedentes de diversos contextos escolares-por ejemplo, algunos profesores tienen un número elevado de aprendices de la lengua inglesa, mientras que otros tienen un número elevado de estudiantes con problemas comportamentales. Estos diversos factores pueden afectar lo que los profesores quieren y son capaces de aprender en las actividades de DPD (e.g., Roschelle et al., 2010).

Esta variación en las respuestas de los profesores al mismo DPD implica que el DPD debería ajustarse a las necesidades individuales de los profesores. Dicho ajuste, logrado al conectar el DPD con las evaluaciones formales de los profesores, es una de las tendencias más importantes en USA en los últimos cinco años aproximadamente (Youngs, 2013). Con frecuencia descrito como "DPD diferenciado", este DPD puede adoptar dos métodos. El primero consiste en ofrecer un catálogo de oportunidades de 
DPD (experiencias online, talleres, lecturas y otras actividades), conectadas con aquellas áreas de la práctica docente evaluadas en las observaciones del profesorado. De este modo, los profesores pueden seleccionar aquellas oportunidades centradas en las áreas donde necesitan mejorar. El segundo método consiste en utilizar datos de las evaluaciones del profesorado en contextos de mentoría y supervisión, lo cual permite a los mentores ajustar su trabajo en función de las fortalezas y debilidades específicas de cada profesor (Allen, Pianta, Gregory, Mikami, \& Lun, 2011). Todavía no sabemos bien cómo el DPD diferenciado está siendo aplicado en la práctica, o si está teniendo los efectos deseados.

Uno de los desafíos importantes es cómo combinar con éxito las ideas de participación colectiva y de DPD diferenciado. El objetivo no es convertir DPD en una experiencia completamente individualizada, sino ofrecer experiencias colectivas que se ajusten a grupos de profesores con necesidades y problemas similares. Por ejemplo, el DPD podría ofrecer diferentes sesiones o actividades para profesores que nunca han realizado una cierta intervención que para aquellos que ya han tenido experiencias previas con ella. Este tipo de DPD diferenciado se puso en práctica en el desarrollo de un programa informático matemático basado en los principios de la ciencia cognitiva, cuyo objetivo era ayudar a estudiantes de sexto grado a aprender sobre fracciones y medidas. A los profesores se les ofreció un DPD diferenciado, en función de su nivel de conocimiento y experiencia con el programa informático. Los resultados mostraron que los profesores se beneficiaron de esta diferenciación (Bowdon, Massey, \& Kregor, 2015).

\section{DPD es más exitoso cuando está explícitamente conectado con la práctica de clase}

Los estudios recientes de DPD han aportando una mejor comprensión sobre los aspectos específicos relativos a la coherencia que son especialmente importantes para el aprendizaje docente. En concreto, hemos encontrado que un aspecto importante de la coherencia es que el DPD esté alineado con el material que los profesores enseñan en clase.

Por ejemplo, Santagata, Kersting, Givvin y Stigler (2011), en un estudio experimental aleatorizado de DPD en matemáticas, alinearon los materiales del DPD con el currículo de los profesores, asegurando que éstos recibían DPD en cada área concreta inmediatamente antes de enseñar dicho área en clase a sus estudiantes. La variación en el ritmo de los profesores interfirió en el estudio, dado que la presentación de contenidos no coincidió en el 50\% de los profesores. Sin embargo, los investigadores encontraron que la implementación y los efectos fueron significativamente mejores para aquellos profesores cuyo ritmo estuvo alineado con el del DPD - es decir, el DPD que estos profesores recibieron estuvo directamente alineado con las lecciones que enseñaron inmediatamente después del DPD. De forma similar, el DPD que Penuel y sus colegas (2011) ofrecieron estaba completa y explícitamente conectado con el currículum que los profesores estaban utilizando para enseñar las ciencias de la tierra a sus 
estudiantes. Los estudiantes de aquellos profesores que participaron en el DPD alcanzaron resultados de aprendizaje significativamente mejores que los de aquellos que no.

Del mismo modo, varios investigadores han atribuido el fracaso de sus programas de DPD a la falta de conexión directa con la práctica de clase de los profesores. Una hipótesis para explicar los decepcionantes resultados de programas de DPD muy intensos y focalizados en el contenido, como aquellos estudiados por Garet y sus colectas $(2009,2011)$, es que el DPD es menos efectivo cuando no ayuda a los profesores a traducir el conocimiento o las estrategias adquiridas en sus lecciones y rutinas de enseñanza diarias.

Estos estudios sugieren que a la hora de diseñar DPD, deberíamos considerar con precaución la facilidad con la que el DPD puede integrarse en las lecciones de los profesores. Debemos afrontar el problema del alineamiento con las lecciones de forma deliberada, y ofrecer a los profesores apoyos, guía y práctica para integrar los nuevos conocimientos o pedagogías en su enseñanza diaria, en vez de abandonarles con esta carga cuando vuelven a clase.

\section{La investigación e implementación del DPD debe considerar las condiciones en áreas urbanas}

La cuarta lección aprendida a partir de estudios experimentales es que tanto en el estudio como en el diseño del DPD deberíamos tener en cuenta ciertas situaciones que se producen con frecuencia en los contextos urbanos de USA. En concreto, la movilidad interna y externa tanto del director, como de los profesores y estudiantes, así como la movilidad interna de los profesores entre distintas asignaturas y niveles educativos. En las escuelas de USA, los profesores cambian frecuentemente de escuela año a año, o se les asigna enseñar diferentes asignaturas o en diferentes niveles. Del mismo modo, los estudiantes cambian de escuela con frecuencia por varias razones, como cuando las familias se trasladan a un barrio diferente dentro del mismo distrito. Los programas de DPD deben ser capaces de responder a la realidad respecto a la movilidad del director, de los profesores y de los alumnos. Por ejemplo, las decisiones relacionadas con la intensidad y la duración del DPD, las oportunidades de re-entrenamiento, o la duración del contacto con los estudiantes durante el año escolar, deberían considerar las fluctuaciones de las poblaciones urbanas y ajustarse a la inestabilidad que, lamentablemente, caracteriza hoy día a la mayoría de las escuelas urbanas en USA (Garet, 2012). Por ejemplo, los programas de formación permanente deberían desarrollar modos para incluir a los profesores que accedan a la escuela en la mitad del año escolar, así como desarrollar procedimientos de análisis de datos que consideren el hecho de que algunos estudiantes sólo están expuestos a ciertas intervenciones durante parte del año escolar, como cuando acceden a la nueva escuela durante el otoño o la primavera. 
Los líderes juegan un rol fundamental apoyando y animando a los profesores para que apliquen las ideas y estrategias aprendidas en DPD en sus clases

Finalmente, la investigación ha demostrado que es más probable que los profesores utilicen las ideas y estrategias aprendidas en DPD cuándo éstas son coherentes con las prioridades de los líderes escolares. Los líderes juegan un papel crucial al ofrecer a los profesores tiempo para participar en DPD y para practicar lo que han aprendido. También pueden jugar un papel importante a la hora de incluir las actividades de DPD en la evaluación de los profesores. El apoyo y entusiasmo de los distritos y de los líderes escolares tienen una influencia decisiva en la habilidad, disponibilidad y motivación de los profesores para probar las nuevas ideas, actividades y currículos facilitados durante DPD (Desimone, 2002). Esta idea es consistente con las teorías sobre el rol que los líderes y los incentivos desempeñan al determinar las acciones del profesor (e.g., Porter, Archbald, \& Tyree, 1990).

\section{Tendencias Actuales en el DPD en USA}

Además de estos recientes resultados de investigación, que contribuyen a nuestra comprensión de cómo mejor diseñar e implementar DPD, existen varias tendencias que están afectando la naturaleza y calidad del DPD en USA.

Una tendencia reciente en USA ha sido el decrecimiento de los talleres y cursos de corta duración, que tradicionalmente han sido muy comunes en USA. En este tipo de talleres y cursos, los profesores se reúnen durante 1-3 horas para escuchar una comunicación sobre un tópico aislado. Los datos nacionales muestran que, durante las dos pasadas décadas, los distritos se han distanciado de este tipo de experiencias, moviéndose hacia DPD que integra las cinco características clave descritas anteriormente (Smith \& Desimone, 2003). Por ejemplo, un análisis de la "Encuesta de Escuelas y Personal" (Schools and Staffing Survey), con carácter representativo a nivel nacional, mostró que menos del $20 \%$ de los profesores en USA participaron en ocho horas o menos de DPD en el año escolar 2011-2012 (U.S. Department of Education, 2012). Otro estudio nacional sobre DPD en USA mostró que el porcentaje de profesores que participaron en DPD relacionado con el contenido disciplinar que enseñaban aumentó del 59\% en el año 2000 al 83\% en 2004, llegando al 87\% en 2008 (Wei, Darling-Hammond, \& Adamson, 2010). Estos resultados sugieren que los proveedores de DPD han comprendido la importancia del DPD continuo y focalizado en el contenido, sentando las bases para que profesores tengan cada día mayor acceso a este tipo de oportunidades.

Otra tendencia, como se mencionó anteriormente, ha sido la creciente conexión entre el DPD y las evaluaciones de los profesores. Esta práctica está cada día más extendida, y está produciendo mejoras en cómo el DPD se organiza, administra y evalúa. Los líderes escolares incluyen en sus evaluaciones anuales cada vez más medidas del grado en que los profesores utilizan en clase el conocimiento y las prácticas aprendidas en DPD. Esto crea un ciclo de política educativa más coherente, donde las actividades de 
DPD en las que los profesores participan están explícitamente valoradas y supervisadas por los líderes escolares, formando parte del sistema de evaluación.

Una tercera nueva dirección en DPD es el creciente uso de videos como herramienta para la observación y la supervisión. Por ejemplo, la disponibilidad de video-tecnologías nuevas y de bajo costo ha hecho posible que un profesor pueda ser grabado dando clase en múltiples ocasiones durante el año escolar, y que un supervisor codifique los videos desde un lugar remoto. Un estudio reciente de Allen y sus colegas (2011) ha mostrado que apoyar y supervisar a los profesores a partir de videos puede tener efectos positivos en sus prácticas docentes y en aprendizaje de los estudiantes.

Otro modo potencialmente interesante de usar videos consiste en ofrecer a los profesores un catálogo de recursos. Por ejemplo, varios estados en USA ofrecen videos gratuitos donde se muestran diversas formas de enseñar contenidos curriculares concretos, de forma que los profesores pueden utilizarlos como recursos a medida que planean sus lecciones diarias (Council of Chief State School Officers, n.d.). Por el momento, no existen datos fiables sobre la frecuencia con que los profesores utilizan dichos recursos ni sobre cuán efectivos son para mejorar las prácticas docentes. Este tema, por tanto, requiere más investigación.

La reciente elaboración de un conjunto de rúbricas de alta calidad para la observación de prácticas docentes, tales como "Calidad de Instrucción en Matemáticas" (Mathematics Quality of Instruction, MQI) (Hill et al., 2008) y "Calidad de Evaluación Instruccional" (Instructional Quality Assessment, IQA) (Junker et al., 2006) han incrementado el potencial uso de los videos de clases. Mediante grabaciones de clases, las administraciones educativas y los investigadores pueden utilizar distintos protocolos para codificar las observaciones de acuerdo múltiples dimensiones, enriqueciendo de este modo el potencial de los datos.

\section{Desafíos}

Los nuevos conocimientos y tendencias en el área del DPD vienen de la mano de varios desafíos. Uno de los principales desafíos del DPD en USA es la tensión producida entre la existencia de numerosos proveedores de DPD y el logro de la coherencia. Como resultado, la experiencia de los profesores en DPD es muchas veces fragmentada, con poca continuidad a lo largo de las distintas actividades, y con falta de un diseño acumulativo. El DPD es patrocinado por muchas agencias. Algunas actividades de DPD son ofrecidas por los estados, otras por los distritos, otras por departamentos universitarios, otras por editoriales, y otras por consultores y organizaciones independientes. El DPD, por tanto, puede tener muy distintos propósitos.

Algunas actividades de DPD son diseñadas para apoyar la aplicación de nuevos currículos o programas; otras para mejorar el conocimiento de los profesores en disciplinas concretas (e.g., algebra, biología); otras son diseñadas para que los profesores aprendan nuevos enfoques pedagógicos (e.g., enseñanza diferenciada); y otras se enfocan en poblaciones muy concretas (e.g., aprendices del inglés como 
segunda lengua). Asimismo, el DPD es financiado y gestionado de modos muy diversos. Pese a la clara inefectividad del modelo de talleres y cursos cortos, algunos distritos escolares continúan ofreciendo este tipo de eventos porque no cuentan con los recursos o la capacidad para diseñar y ofrecer actividades de DPD que resulten más coherentes e integradoras para el profesorado. Además, a las escuelas y distritos les resulta difícil obtener información precisa y completa de las distintas actividades de DPD disponibles, o de las experiencias previas de los profesores en dichas actividades (Wilson, Rozelle, \& Mikeska, 2011).

Esta multiplicidad en los tipos de DPD también hace difícil aprender de las investigaciones. Es decir, el DPD varía en tantos aspectos que es complicado llegar a conclusiones sobre qué factores contribuyen su éxito o fracaso. Es necesario manipular características muy específicas del DPD para aislar su influencia (véase Penuel et al., 2011). Otro desafío en el estudio del DPD es que el seguimiento del profesorado en diferentes actividades de DPD no se realiza con rigor en la mayoría de distritos. Si los distritos hicieran un mejor trabajo en la supervisión y registro de las actividades de DPD en que los profesores participan, los datos podrían ser utilizados en sistemas longitudinales de datos administrativos para ayudar a identificar patrones de participación, y eventualmente conectar dichos patrones con los resultados de los profesores y los estudiantes.

Otro desafío importante, como ya se discutió más arriba, es ser explícito sobre lo que se espera que los profesores aprendan de las actividades de DPD. En muchos casos, la teoría de la acción necesita ser articulada con mucha mayor claridad. Por ejemplo, pensando en DPD diseñado para ayudar a los profesores a incrementar su conocimiento disciplinar, ¿cómo se espera que traduzcan dicho conocimiento en clase, de forma que beneficie el aprendizaje de los estudiantes? Algunos estudios han mostrado que la conexión explícita de las ideas o conductas enseñadas en DPD con los libros, las lecciones, o los materiales que los profesores utilizan en clase es, en muchos casos, una pieza muy poderosa y quizás necesaria del puzle (e.g., Santagata et al., 2011).

\section{Siguientes Pasos}

En el contexto de todas estas ideas y desafíos, existen varias razones para pensar que la investigación y el desarrollo del DPD continuará avanzando en la siguiente década. En primer lugar, en USA se han desarrollado nuevas medidas de los resultados del profesorado. En concreto, hoy contamos al menos con algunas medidas del conocimiento disciplinar del profesorado (e.g., Hill, Rowan, \& Ball, 2005). En segundo lugar, la creciente disponibilidad de video-tecnología puede simplificar el análisis de las relaciones entre el DPD y la mejora de las prácticas docentes.

En tercer lugar, la creciente disponibilidad de medidas de valor añadido del profesorado hace posible examinar las relaciones entre su participación en DPD y el cambio en su efectividad (al menos en relación a los resultados de los estudiantes en pruebas estandarizadas). La disponibilidad de estos tipos de medidas permitirían la 
realización de estudios cuasi-experimentales. Sin embargo, el principal reto aquí será obtener datos relativos a la participación en DPD de los profesores, dada la naturaleza fragmentaria de las oportunidades que generalmente se ofrece a los profesores, además de que existen pocos distritos que recojan datos de participación en DPD de forma sistemática. Algunos estados y distritos han comenzado a catalogar la participación de los profesores en DPD, de forma que estos datos podría ser conectados con datos longitudinales de resultados académicos en muchos estados.

Finalmente, existe un potencial creciente para llevar a cabo rápidos estudios experimentales al implementar nuevas oportunidades de DPD, que permitan revisar y modificar su diseño. Por ejemplo, si un distrito planea ofrecer DPD a profesores con necesidades específicas, identificadas a partir del sistema de evaluación docente, en el primer año algunos profesores podrían ser asignados para recibir DPD intensivo, y los resultados de su evaluación podrían ser cuidadosamente analizados. Este tipo de investigación permitiría obtener resultados más rápidamente que en los típicos estudios experimentales, que duran aproximadamente 1-3 años.

\section{Conclusión}

Existe sólida evidencia de que las cinco características centrales del DPD efectivo han sido adoptadas como "mejores prácticas" en numerosos países (Kennedy, 2014), y de que los profesores de otros países desarrollados tienen desafíos y problemas similares a los experimentados por los profesores en USA, como hemos descrito aquí (e.g., Jones, 2011; O'Brien, 2011). Sin embargo, es necesario realizar más trabajo para llevar la investigación en DPD al siguiente nivel, en particular para traducir las cinco características generales en actividades concretas que sean efectivas en distintos contextos. También necesitamos más información sobre los aspectos específicos de las cinco características que resultan importantes en diferentes contextos, con objeto de formar una mejor comprensión de por qué ciertas experiencias de DPD funcionan y otras no.

En términos de diseñar y ofrecer DPD de alta calidad, resulta esencial establecer bucles periódicos de supervisión y evaluación. Dichos bucles pueden ayudar a los proveedores del DPD, tanto si son escuelas como distritos u otros, a crear círculos de mejora continuos. Además, esto permite abordar las debilidades identificadas en cualquiera de las cinco características centrales. Otro área que nos gustaría enfatizar es la importancia de incluir un riguroso componente evaluador en el DPD, de modo que podamos continuar incrementando nuestra comprensión de por qué ciertas actividades de DPD son efectivas y otras no.

\section{Referencias}

Allen, J. P., Pianta, R. C., Gregory, A., Mikami, A. Y., \& Lun, J. (2011). An interaction-based approach to enhancing secondary school instruction and student achievement. Science, $333,1034-1037$. 
Bowdon, J., Massey, C., \& Kregor, J. (2015). Unpacking implementation of a mathematicsbased software intervention. Philadelphia, PA: Cognitive Science Center, University of Pennsylvania.

Cohen, D., \& Ball, D. L. (1990). Relations between policy and practice: A commentary. Educational Evaluation and Policy Analysis, 12(3), 249-256.

Council of Chief State School Officers. (n.d.). http://www.ccsso.org/resources/digital_resources/common_core_implementation_video series.html

Desimone, L. (2002). How can comprehensive school reform models be successfully implemented? Review of Educational Research, 72(3), 433-479.

Desimone, L. M. (2009). Improving impact studies of teachers' professional development: Toward better conceptualizations and measures. Educational Researcher, 38(3), 181199.

Desimone, L., Porter, A. C., Garet, M., Yoon, K., \& Birman, B. (2002). Effects of professional development on teachers' instruction: Results from a three-year study. Educational Evaluation and Policy Analysis, 24(2), 81-112.

Desimone, L., Smith, T., \& Phillips, K. (2013). Linking student achievement growth to professional development participation and changes in instruction: A longitudinal study of elementary students and teachers in Title I schools. Teachers College Record, 115(5), $1-46$.

Desimone, L., \& Stuckey, D. (2014). Sustaining professional development. In. L. Martin, S. Kragler, D. Quatroche, \& K. Bauserman (Eds.), Handbook of professional development in education: Successful models and practices, prek-12 (pp. 467-482). New York, NY: Guilford Publications.

Garet, M. (2012, March). Recommendations for professional development. In Supporting implementation of the Common Core State Standards for mathematics (pp. 59-60). Washington, DC: CCSSO and North Carolina State University.

Garet, M. S., Cronen, S., Eaton, M., Kurki, A., Ludwig, M., Jones, W., Uekawa, K., ... Silverberg, M. (2008). The impact of two professional development interventions on early reading instruction and achievement. Washington, DC: National Center for Education Evaluation and Regional Assistance, Institute of Education Sciences, U.S. Department of Education.

Garet, M., Porter, A., Desimone, L., Birman, B., \& Yoon, K. (2001). What makes professional development effective? Analysis of a national sample of teachers. American Education Research Journal, 38(3), 915-945.

Garet, M., Wayne, A., Stancavage, F., Taylor, J., Eaton, M., Walters, K., \& Doolittle, F. (2011). Middle school mathematics professional development impact study: Findings after the second year of implementation (NCEE 2011-4024). Washington, DC: National Center for Education Evaluation and Regional Assistance, Institute of Education Sciences, U.S. Department of Education.

Garet, M. S., Wayne, A. J., Stancavage, F., Taylor, J., Walters, K., Song, M., \& Doolittle, F. (2010). Middle school mathematics professional development impact study: Findings after the first year of implementation (NCEE 2010-4009). Washington, DC: National 
Center for Education Evaluation and Regional Assistance, Institute of Education Sciences, U.S. Department of Education.

Gersten, R., Dimino, J., Jayanthi, M., Kim, J. S., \& Santoro, L. E. (2010). Teacher study group: Impact of the professional development model on reading instruction and student outcomes in first grade classrooms. American Educational Research Journal, 47(3), 694-739.

Hill, H. C., Rowan, B., \& Ball, D. L. (2005). Effects of teachers' mathematical knowledge for teaching on student achievement. American Educational Research Journal, 42(2), 371406. doi: $10.3102 / 00028312042002371$

Hill, H. C., Blunk, M., Charalambous, C., Lewis, J., Phelps, G. C., Sleep, L., \& Ball, D. L. (2008). Mathematical knowledge for teaching and the mathematical quality of instruction: An exploratory study. Cognition and Instruction, 26, 430-511.

Jones, K. (2011). Central, local and individual continuing professional development (CPD) priorities: Changing policies of CPD in Wales. Professional Development in Education, 37(5), 759-776.

Junker, B. W., Matsumura, L. C., Crosson, A., Wolf, M. K., Levison, A., Wiesberg, J., \& Resnick, L. (2006). Overview of the Instructional Quality Assessment (CSE Technical Report 671). Los Angeles, CA: University of California, National Center for Research on Evaluation, Standards, and Student Testing.

Kennedy, A. (2014). Understanding continuing professional development. Professional Development in Education, 40(5), 688-697.

O'Brien, J. (2011). Continuing professional development for Scottish teachers-tensions in policy and practice. Professional Development in Education, 37(5), 777-792.

Penuel, W. R., Gallagher, L. P., \& Moorthy, S. (2011). Preparing teachers to design sequences of instruction in earth systems science: A comparison of three professional development programs. American Educational Research Journal, 48(4), 996-1025.

Piasta, S. B., Dynia, J. M., Justice, L. M., Pentimonti, J. M., Kaderavek, J. N., \& Schatschneider, C. (2010). Impact of professional development on preschool teachers' print references during shared read alouds: A latent growth curve analysis. Journal of Research on Educational Effectiveness, 3(4), 343-380.

Porter, A., Archbald, D., \& Tyree, Jr., A. (1990). Reforming the curriculum: Will empowerment policies replace control? In S. H. Fuhrman \& B. Malen (Eds.), The politics of curriculum and testing: The 1990 yearbook of the Politics of Education Association (pp. 11-36). New York: Falmer.

Roschelle, J., Shechtman, N., Tatar, D., Hegedus, S., Hopkins, B., Empson, S., Knudsen, J., \& Gallagher, L. P. (2010). Integration of technology, curriculum, and professional development for advancing middle school mathematics: Three large-scale studies. American Educational Research Journal, 47(4), 833-878.

Sailors, M., \& Price, L. R. (2010). Professional development that supports the teaching of cognitive reading strategy instruction. The Elementary School Journal, 110(3), 301-322.

Santagata, R., Kersting, N., Givvin, K. B., \& Stigler, J. W. (2012). Problem implementation as a lever for change: An experimental study of the effects of professional development program on students' mathematics learning. Journal of Research on Educational Effectiveness, 4(1), 1-24. doi: 10.1080/19345747.2010.498562 
Smith, T., \& Desimone, L. (2003). Do changes in patterns of participation in teachers' professional development reflect the goals of standards-based reform? Educational Horizons, 81(3), 119-129.

Smith, T. M., Desimone, L. M., \& Ueno, K. (2005). "Highly qualified" to do what? The relationship between NCLB teacher quality mandates and the use of reform-oriented instruction in middle school math. Educational Evaluation and Policy Analysis, 27(1), 75-109.

U.S. Department of Education. (2012). National Center for Education Statistics, Schools and Staffing Survey (SASS), Public and Private Teachers Data File.

Wayne, A. J., Yoon, K. W., Zhu, P., Cronen, S., \& Garet, M. S. (2008). Experimenting with teacher professional development: Motives and methods. Educational Researcher, 37, 469-279. doi: 10.3102/0013189X08327154

Wei, R. C., Darling-Hammond, L., \& Adamson, F. (2010). Professional development in the United States: Trends and challenges. Dallas, TX: National Staff Development Council.

Wilson, S. M., Rozelle, J. J., \& Mikeska, J. N. (2011). Cacophony or embarrassment of riches: Building a system of support for quality teaching. Journal of Teacher Education, 62, 383-394

Youngs, P. (2013). Using teacher evaluation reform and professional development to support Common Core assessments. Washington, DC: Center for American Progress. Retrieved from http://cdn.americanprogress.org/wp-

content/uploads/2013/01/YoungsCommonCore.pdf 